\title{
Experiment study of using parallel plate sensor for the detection of conductive material
}

\author{
N. Lokmanulhakim, W. N. Abd Rashid, N. H. Hussin, M. F. Mukhtar \\ Department of Electronic and Computer Engineering Technology, Universiti Teknikal Malaysia Melaka, Malaysia
}

\begin{tabular}{l}
\hline \hline Article Info \\
\hline Article history: \\
Received Jan 11, 2019 \\
Revised Jul 23, 2019 \\
Accepted Jul 30, 2019 \\
\hline
\end{tabular}

\section{Keywords:}

Conductive material

Electrical capacitance

Gold

Parallel plate sensor

Square electrode

Tomography

\begin{abstract}
Gold is one of the precious metal that has a good demand market in the world. Due to that, many fraud cases happen especially when dealing with the jewelry. Thus, verifying gold content is important and need to be done with the best techniques. This paper introduces an ECT sensor of two parallel plates using square electrodes. This sensor was investigated either it capable to detect the conductive materials; Gold. A complete architecture of a system is designed which include the sensor design, the main controller unit design and graphic user interface (GUI). A two-plate ECT sensor with 2 electrodes each plate will fabricate on the printed circuit board (PCB). This experiment will implement single electrode excitation method. When electrode 1 was injected by the source, electrodes other than electrode 1 are at the virtual earth potential or called as detecting electrode. Independent capacitance measurements were taken from the three electrodes with different excitation frequency. The frequency will vary from $50 \mathrm{~Hz}$ to $9.5 \mathrm{MHz}$. The experiment result shows that the two parallel plate ECT sensor is able to measure the conductive materials.
\end{abstract}

Copyright $(0) 2019$ Institute of Advanced Engineering and Science. All rights reserved.

\section{Corresponding Author:}

Nor Hafizah Binti Hussin,

Department of Electric Engineering Technology,

Universiti Teknikal Malaysia Melaka,

Hang Tuah Jaya, 76100 Durian Tunggal, Melaka, Malaysia.

Email: norhafizah.hussin@utem.edu.my

\section{INTRODUCTION}

Gold is the yellow precious metal that has been used in many applications likes medicine, electronics, jewelry, investment, and others. Thus, gold is one of the commodities which has high demand in the global market especially in jewelry industries. More than $75 \%$ of gold output was processed into jewelry. The misrepresentation of gold content has been a source of concern at all levels of the industries. In other to secure the purity of gold and to protect the retailer and consumer from fraud, the detection of gold is very important.

There are a number of techniques have evolved over the years for determining gold content either using the ancient or modern technique. Cupellation (fire assay) [1] is one of the traditional methods that has been used worldwide for gold determination and it still remains the most accurate method.Hence, it was accepted to become the standard technique to be compared and covered in the ISO 11426:1993 [2]. However, this procedure is a non-destructive method because sample being destroyed as it should be melted [3]. For the modern technique, there are few analytical methods that been practice for assay gold such as X-ray fluorescence (XRF). XRF is the most popular nuclear method that is used to varied gold but this technique had limited to testing the surface and its ability about the inner details of test samples remains unknown [4], [5]. The other techniques that usually practic for varifying gold is Inductive Couple Plasma (ICP) [6, 7], Atomic Absorption Spectrometry (AAS) [8, 9], Computed Tomography, and the latest invention is using Dual Energy of Micro-Computed Tomography. 
Each technique have their own advantages, nevertheless for an ideal method of assaying gold could consider few factors such as reliable, simple, rapid, inexpensive, and also non-destructive [10]. Non- destructive test (NDT) is one of the important factors for ensuring that the fineness of gold can be maintained [11, 12]. More recent attention has focused on the application of Electrical Capacitance Tomography (ECT) technique to examine the contents of gold jewellery. Hisyam et al (2016), have studied on developing a new technique using the parallel plate Electrical Capacitance Tomography to check the content of gold bar which promising result to assay the purity and fineness of gold [13]. ECT was known as one of the most developed tomographic modalities among many industrial process tomography techniques This is because, ECT techniques are non-radioactive, non-intrusive and non-invasive, high imaging speed and low cost $[14,15]$. Other than that, ECT also has implement in many industrial applications, including the flow measurement in the oil pipelines [16, 17], wet gas separators, fluidized beds [18, 19], pneumatic conveyors and gas/solids cyclones [20,21]. This shown that ECT was a mature technique and it can be applied in various application. Therefore, the writer would like to purpose to use the ECT technique for verifying the conductive materials. The objective of this paper is to identify the effectiveness of using a parallel plate sensor method for the measurement of the conductive material such as gold bar, aluminium, and iron.

\section{FUNDAMENTAL OF ECT}

The basic concept of ECT is to measure variations of capacitance between electrode pairs, then it will generate a cross-sectional image. This cross-sectional image will represent the permittivity distribution and material distribution [22-24]. Figure 1 describes the whole system of ECT which is consist of three main units:

- The sensor with multi-electrodes,

- The capacitance measuring circuit, and

- A computer for hardware control and data processing, including image reconstruction.

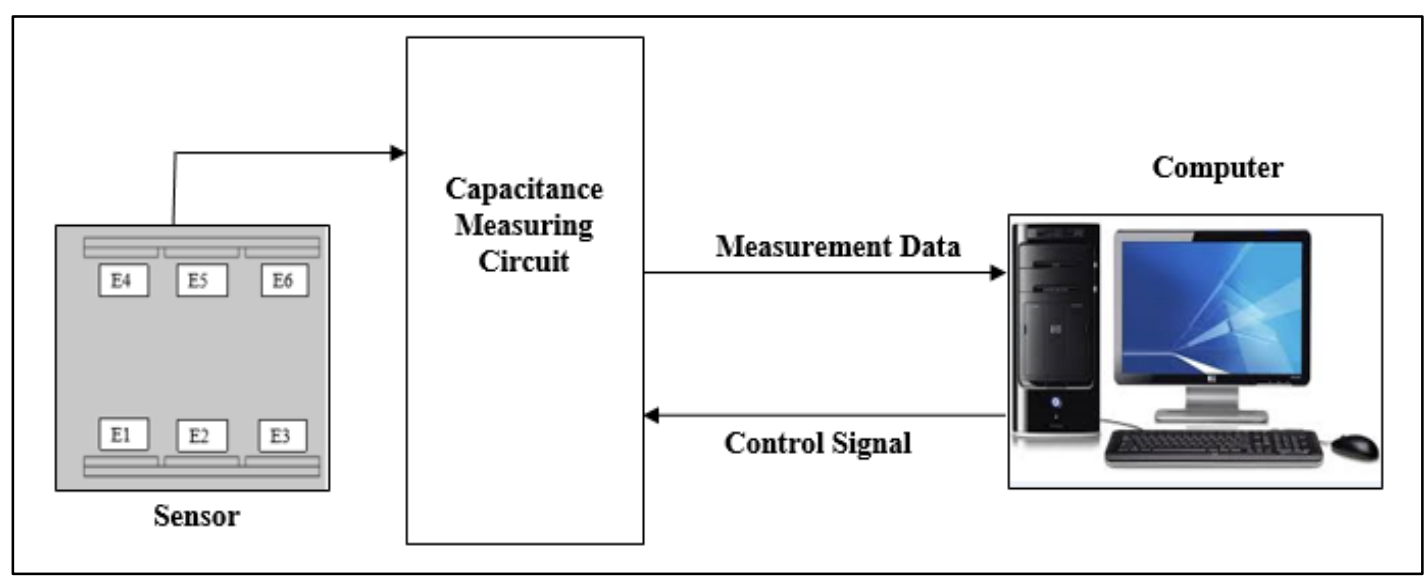

Figure 1. Overview of the ECT system

Figure 1 shows a segmented ECT system with a sensor comprising of 6 electrodes, a capacitance measurement circuit, a central control unit, and a control PC. The electrode which is normally built from a conductive plate acts as the sensing surface that has a direct connection to the measuring area. The electrode provides excitation signals and converts capacitances into voltage signals, which are then conditioned and digitized for data acquisition. The capacitance measuring circuit, known as the signal conditioning circuit, is used to collect data and to convert measurement readings into digitized results. The central control unit synchronizes all operations and facilitates the transfer of data to the control PC. The control PC receives the measurement readings, stores the acquired data, and visualizes reconstructed images.

For ECT system there are two basic problems so- called as forward and inverse problem. The forward problem is to determine inter-electrode capacitances from the permittivity distribution [25, 26]. While the inverse problem is to determine the permittivity distribution from capacitance measurements. 
In this case, the author chooses to use finite-element method (FEM) for solving the forward problem. The relationship between capacitance and permittivity distribution can be expressed as:

$$
C=\frac{Q}{V}=-\frac{1}{V} \iint \varepsilon(x, y) \nabla \emptyset(x, y) d \Gamma
$$

Where $V$ is the potential difference between two electrode, $\varepsilon(x, y)$ is the permittivity distribution in the sensing field, $\varnothing(x, y)$ is the potential distribution and $\Gamma$ is the electrode surface.

Equation (1) can be simplified in some cases. For example, for an ideal parallel -plate sensor with a homogeneous permittivity distribution, it becomes

$$
C=\varepsilon_{o} \varepsilon_{r} \frac{A}{d}
$$

Where $\varepsilon_{o}$ is the permittivity of vacuum $\left(8.84 \times 10^{-12}\right), \varepsilon_{r}$ is the relative permittivity of the material inside the sensor, A is the area of the plates and $d$ is the distance between the two plates. Equation (2) implies such a simple relationship that capacitance is proportional to permittivity.

\subsection{Electrode sensor design}

ECT sensors are formed by an array of the electrode which typically consists of 8, 12, 16 electrodes in the typical ECT, normally the electrodes are a circular shape in order to encircle the circular type of pipelines. Anyhow, in some specific application such as industrial boiler, square shape ECT sensor are needed. The advantage of the square shape it can improve image quality in the central region compared with the circular sensor [27]. Almost all of ECT sensors is fixed and installed on the pipeline with an enclosing sensing area. Thus it is limited to some application that has irregular shapes and open sensing area. Therefore for this experiment, the writer will use square parallel plate sensor which allows the sensors to be fixes and assembled independently by following the shape of the material tested.

In this study, there are few key issues for designing the ECT sensors need to be considered. First are the number and length of the electrodes. For this experiment, 4 electrodes will be used. The length also just maximum $21 \mathrm{~mm}$ because follow the material tested. However, Yang et al (2010) stated that the electrodes cannot be too short because of the limit in the resolution of the capacitance measuring circuits and the fringe effect. Second is the value of the standing capacitance. There are few factors that can influence the value of standing capacitance to include the thickness and permittivity of the material, the distance between two plate and angle of elevation of the parallel plate. Other factors that can cause standing capacitance is earthed screen and driver guard electrodes.

For fabricated the electrodes, the material used must be a highly conductive material, such as copper, aluminum, silver, steel, tungsten or iron. Table 1 shows a comparison between some high conductivity materials. Therefore, for this experiment writer decide to fabricate electrode using copper as the material. This is because, copper can easily be found on any Printed Board (PCB) moreover it also highly conductive material, easy to be fabricated and also low cost.

Table 1 . The comparison of electrical conductivity between high conductivity materials

\begin{tabular}{ll}
\hline Material & Electrical Conductivity $\sigma(\mathrm{S} / \mathrm{m})$ at $20^{\circ} \mathrm{C}$ \\
\hline Silver & $6.30 \times 10^{7}$ \\
Copper & $5.98 \times 10^{7}$ \\
Aluminum & $3.5 \times 10^{7}$ \\
Iron & $1.04 \times 10^{7}$ \\
Zinc & $1.682 \times 10^{7}$ \\
\hline
\end{tabular}

In this experiment, two sensor plates will be placed in parallel. Each sensor consists of either 2 electrodes. The design parameters of the sensors are shown in Figure 2. The configurations of the sensor are:

- Length of shielding layers and wall: $21 \mathrm{~mm}$

- Electrode length: sensor $1=18 \mathrm{~mm}$

- Gap between adjacent electrodes: $1 \mathrm{~mm}$

- Material of shielding layers and electrode: Copper 


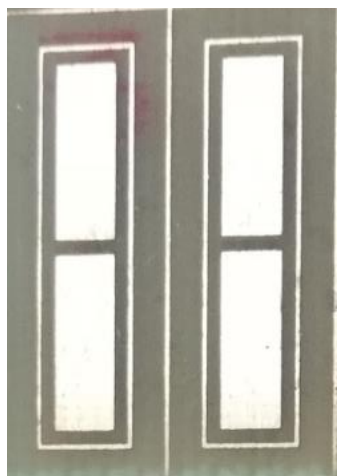

Figure 2. Sensor with 4 electrodes

\subsection{Main control unit design}

The signal conditioning circuit consists of few parts; a capacitance measuring circuit, amplifier circuit, absolute circuit, low pass circuit and also analog to digital converter. At the first stage, a sine wave voltage $\mathrm{V}_{\mathrm{i}}$ is supplied from the function generator with a frequency of $500 \mathrm{kHz}$ and amplitude $5 \mathrm{Vpk}$-pk. The excitation voltage $V_{i}$ is applied to the unknown capacitance $C_{x}$. A wide bandwidth Op-Amp, with feedback capacitance $C_{f}$ and feedback resistance $R_{f}$ converts the current into an AC voltage, $V_{0}$ given by:

$$
V o=\frac{j \omega C_{x} R_{f}}{j \omega C_{f} R_{f}+1} V i
$$

The angular frequency $(\omega)$ is from sine wave source. Then the capacitance feedback is selected to be dominant equation (3) becomes:

$$
V o=-\frac{C_{x}}{C_{f}} V_{i}
$$

This AC signal is amplified further by an AC amplifier to accommodate a large range of capacitance values. Figure 3 shows AC-based capacitance measurement circuit.

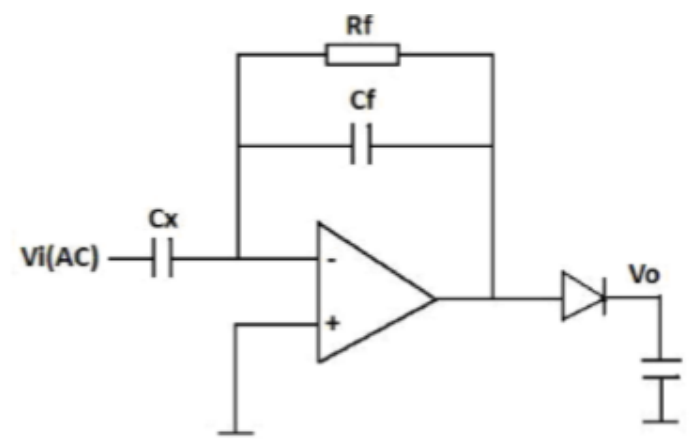

Figure 3. AC-based capacitance measurement circuit [23]

The writer added the application of absolute circuit for processes the output voltage to be equal to the input voltage regardless of polarity. In other words, both polarities of the input signal produce the same polarity as the output signal. Later the absolute value circuit will be combined with the first order low-pass filter to reduce noise and to get a clean DC output from the AC input voltage. NI MyRio was chosen as the main controller which will synchronize the whole operation of the system. Moreover, the data in an analog form which has been collected from the sensor will be transferred to the Myrio which act as the analog to digital converter. Lastly, GUI was being interfaced with the controller module to record the analogue inputs and calculate the average capacitance between the electrodes. The block diagram in Figure 4 shows the whole process of the ECT system. 


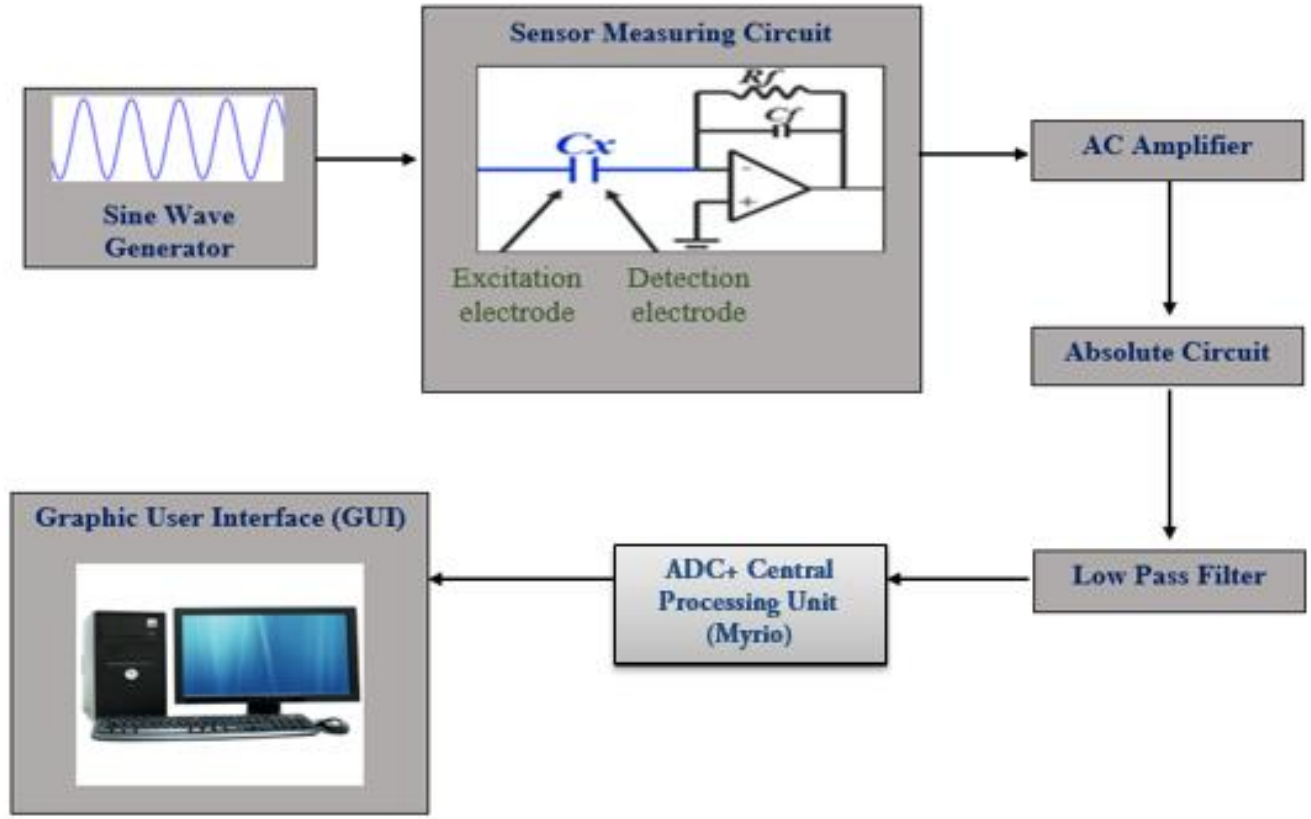

Figure 4. Block diagram of electrical capacitance tomography system

\section{RESULTS AND DISCUSSION}

The experiment was carried out by means of an ECT system for measure capacitance of conductive material. At the early stages three materials were tested; gold bar, iron, and aluminium. The experiment was carried out using a $2 \times 2$ array of electrodes: electrode 1 (E1), electrode 2 (E2), electrode 3 (E3) and electrode 4 (E4). The distance between the parallel plates is fixed to $1 \mathrm{~mm}$. The material will be placed in the middle of the plates. For this system, the traditional method of single electrode excitation was used. First electrode (E1) become the excite electrode or source electrode which is supplied by a sine wave, while the other three electrodes will act as a receiver. These three electrodes will become grounded. In this time, when electrode 1 was injected by the source, electrodes other than electrode 1 are at the virtual earth potential imposed by the transducer and they are called the detecting electrode. Independent capacitance measurements were taken from the three electrodes with different excitation frequency. The frequency will vary from $50 \mathrm{~Hz}$ to $9.5 \mathrm{MHz}$. For these cases, there are 3 independent capacitance measurements among all the combination pairs of electrodes i.e E1-E2, E1-E3, and E1-E4. The data as shown in Figure 5-8.

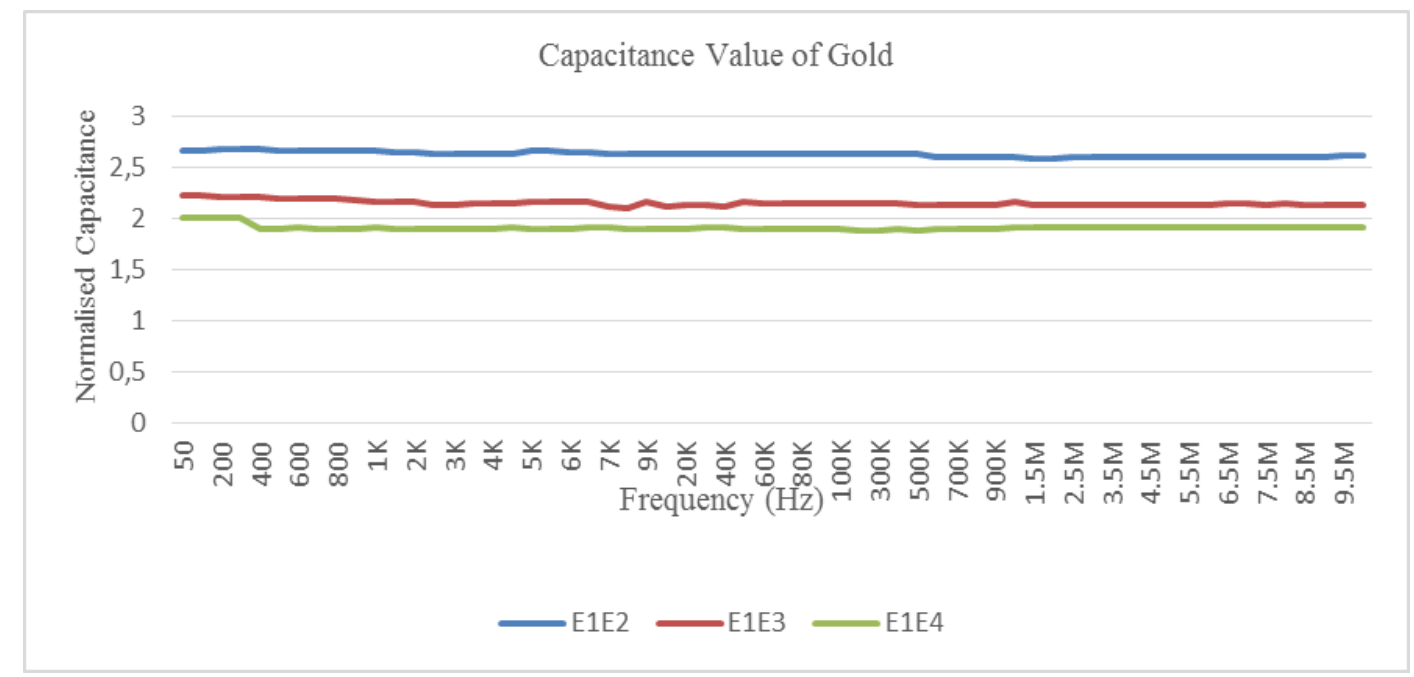

Figure 5. Capacitance value for gold 


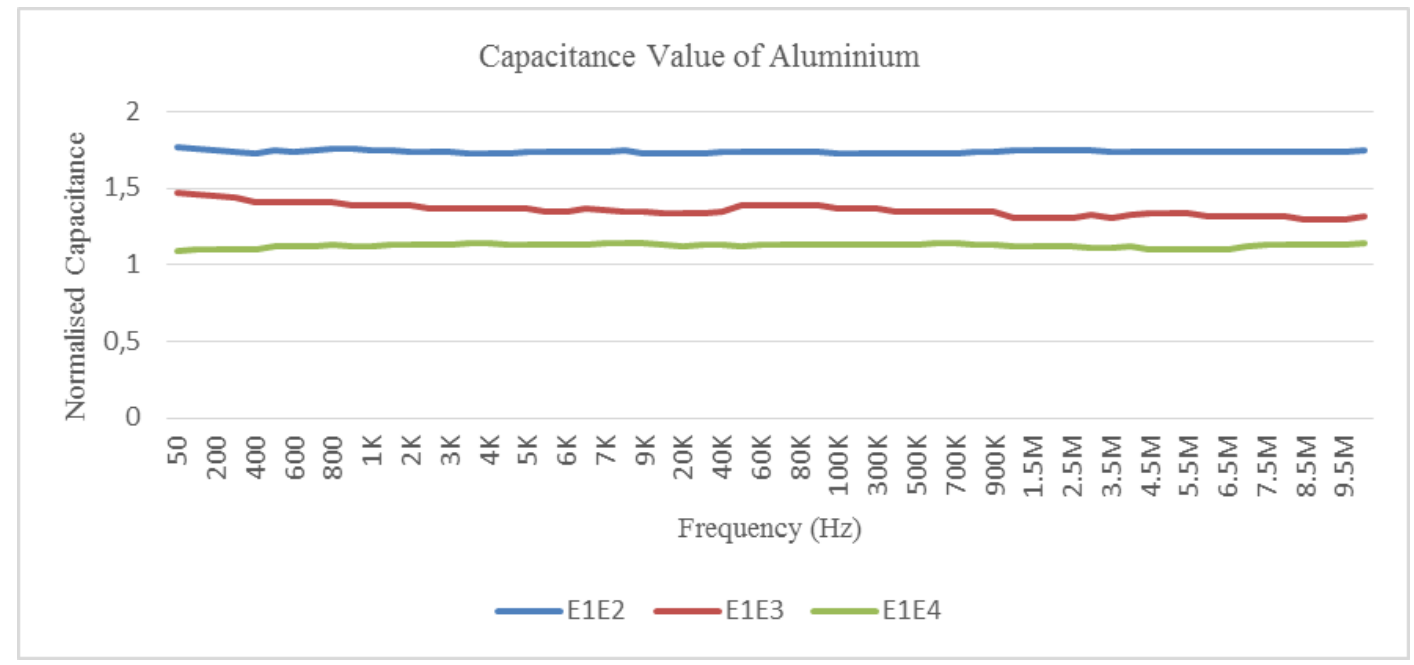

Figure 6. Capacitance value for aluminium

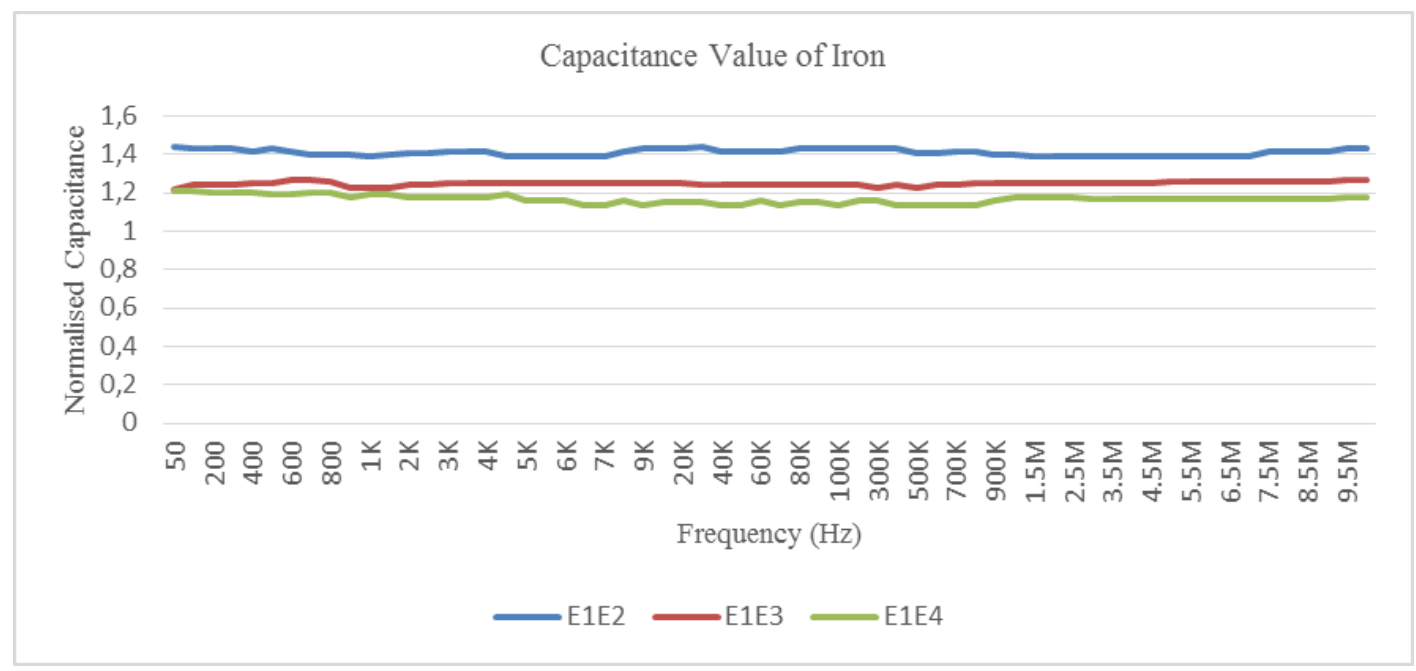

Figure 7. Capacitance value for iron

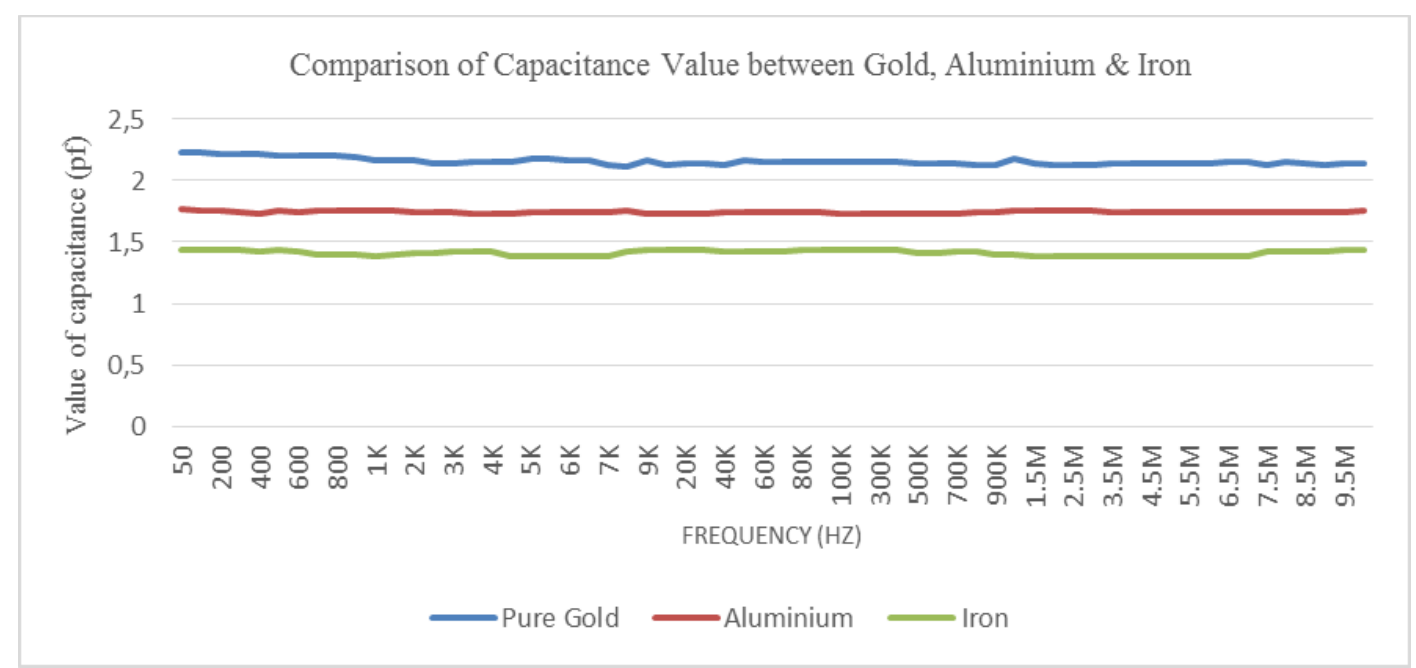

Figure 8. Comparison of capacitance value between pure gold, aluminium and iron 
As shown in the respective results, the measured capacitance is very small due to the small electrodes. The result might be influenced by undesired standing capacitance from the cable and surrounding air. By comparing all materials, the larger capacitance was produced by the pure gold followed by aluminium and iron. This can be related to the electrical conductivity of the materials. The gold state is the highest electrical conductivity $\left(4.52 \times 10^{7} \mathrm{~S} / \mathrm{m}\right)$, aluminium $\left(3.5 \times 10^{7} \mathrm{~S} / \mathrm{m}\right)$ and iron $\left(1.04 \times 10^{7} \mathrm{~S} / \mathrm{m}\right)$. Besides that, the measurement also slightly difference caused by the thickness of the materials. From the observed results, the value measured by electrode E1-E2 was found to produce the highest value among the other combination pairs of electrodes E1-E3 and E1-E4.

\section{CONCLUSION AND FUTURE WORK}

As summarized, the parallel plate sensor is capable to use for verifying the conductive material. The measurement data is consistent for each electrode. However, the value of capacitance is too small. Thus, instead of using traditional single electrode excitation, a multiple-electrode excitation strategy can be adopted. Multiple electrodes will excitation simultaneously. For example, a 2 electrode sensor can be implemented by combining the electrode E1 and E3. With multiple-electrode excitation, the measured capacitance is three times larger than single-electrode excitation [28]. Since this experiment is in the early stages thus some specific issues need to be considered. First is about the effectiveness of the sensing area. Too small or too large sensing area can cause a too high sensitivity near the edge or too low sensitivity will in the central region will produce a distortion. Thus it is recommended to set the length of the shielding layer is equal to the width of the sensing area and also the distance between the two plates [29]. Second, the distance between the two plates. In theoretically, when the distance is changed it will affect the sensitivity. In this experiment, the distance between the two plates is smaller which is good for sensitivity in the central region. However, it can cause the plates to touch the materials. This is because each material have difference thickness. Thus, the suitable distance needs to study more. Third, the angle between the two plates. In some case, the two plates or material tested may not be placed exactly in parallel. The biggest issues for ECT sensor is a number of electrodes which it determines the number of impedance capacitance. The higher image resolution can be produced by the larger impedance measurement. But, if the number of electrodes increases then it will cause a smaller sensing area. With the small sensing area, the measurement is difficult to take. Thus, the best number of the electrode must be investigated relevant with material tested. For the future work, writer propose to focus on the relationship between the electrical conductivity of conductive material with the value of capacitance. The others possible work can be a focus on the image reconstruction.

\section{ACKNOWLEDGMENTS}

Authors are grateful to the financial support by PJP/2018/FTK (15A)/S01641 and Universiti Teknikal Malaysia Melaka (UTeM). We would like to thank our colleagues from the Centre for Telecommunication Research \& Innovation (CeTRI), University Teknikal Malaysia Melaka. Thanks to staff and members of UTeM who give assistance and commitment to me and guide me in various perspective throughout the project. Special thanks must be given to my friends for ideas and comment regarding to my project.

\section{REFERENCES}

[1] K. M. Blyth, et al., "Analysis of Gold Ores by Fire Assay,” J. Chem. Educ., vol. 81, pp. 1780-1782, 2004.

[2] B. C. W. Corti, "Assaying of Gold Jewelry - Ancient And Modern," St. Fe Symp. Jewel. Manuf. Technol., pp. 49-70, 2001.

[3] S. Caporali, et al., "Determination of gold in alloys via potentiometric titration; an alternative to the fire assay," Gold Bull., vol. 43, pp. 122-130, 2010.

[4] A. J. López, et al., "Application of energy-dispersive X-ray fluorescence to jewellery samples determining gold and silver," Gold Bull., vol. 39, pp. 16-21, 2006.

[5] P. Jalas, et al., "XRF Analysis of jewelry using fully standardless fundamental parameter approach," Gold Technol., vol. 35, pp. 28-34, 2002.

[6] K. Pyrzyńska, "Recent developments in the determination of gold by atomic spectrometry techniques," Spectrochim. Acta - Part B At. Spectrosc., vol. 60, pp. 1316-1322, 2005.

[7] J. Abdullah, et al., "Non-destructive assaying gold jewellery using dual-energy micro-computed tomography," J. Teknol., vol. 73, pp. 25-28, 2015.

[8] H. Ashkenani and M. Ali, "Use of ionic liquid in simultaneous microextraction procedure for determination of gold and silver by ETAAS," Microchem. J., vol. 103, pp. 185-190, 2012.

[9] S. Tong, et al., "Determination of gold ( III ) and palladium ( II ) in mine samples by cloud point extraction 
preconcentration coupled with flame atomic absorption spectrometry," pp. 95-102, 2011.

[10] N. Singh, "A rugged, precise and accurate new gravimetry method for the determination of gold: an alternative to fire assay method," Singh SpringerPlus, vol. 1, pp. 1-6, 2012.

[11] W.N. Abd Rashid, et al., "Design Of Non Destructive Testing On Composite Material Using Parallel Plate Electrical Capacitance Tomography: A Conceptual Framework," vol. 79(5-2), pp. 71-76, 2017.

[12] W. Norhisyam, et al., "Development and Experimental Study of Miniature Two-plate Electrical Capacitance Sensor to Detect Dielectric Material," Journal of Telecommunication, Electronic and Computer Engineering (JTEC), vol. 10, pp. 5-10.

[13] W. N. Abd Rashid, et al., "Design of non-destructive test on gold metal using parallel plate capacitance sensor: A conceptual framework," Res. Dev. (SCOReD), 2016 IEEE Student Conf., 2016, pp. 1-6.

[14] Wan Norhisyam Abd, et al., "Electrical capacitance tomography: a review on portable ECT system and hardware design," in Sensor Review, vol. 36(1), pp. 64-70, 2016.

[15] Z. Fan and R. X. Gao, "Enhancement of measurement efficiency for electrical capacitance tomography," IEEE Trans. Instrum. Meas., vol. 60, pp. 1699-1708, 2011.

[16] E. J. Mohamad and R. A. Rahim, "Multiphase Flow Reconstruction in Oil pipelines by Portable Capacitance Tomography," SENSORS, 2010 IEEE, pp. 273-278, 2010.

[17] P. W. Wang, et al., "An ECT / ERT Dual-Modality Sensor for Oil-Water Two-phase Flow Measurement," AIP Conference Proceedings 1592, vol. 3, 2014.

[18] H. G. Wang and W. Q. Yang, "Measurement of fl uidised bed dryer by different frequency and different normalisation methods with electrical capacitance tomography," Powder Technol., vol. 199, pp. 60-69, 2010.

[19] A. Hunt, "Weighing without Touching: Applying Electrical Capacitance Tomography to Mass Flowrate Measurement in Multiphase Flows," SAGE Journal, vol. 47, pp. 19-25, 2015.

[20] W. Zhang, et al., "Application of electrical capacitance tomography in particulate process measurement-A review," Adv. Powder Technol., vol. 25, pp. 174-188, 2014.

[21] A. J. Jaworski and T. Dyakowski, "Application of Electrical Capacitance Tomography for Measurement of GasSolids Flow Characteristics in a Pneumatic Conveying System," Measurement Science and Technology vol. 12(8), pp. 1109, Jul 2001.

[22] Z. Cui, et al., "Electrical Capacitance Tomography With Differential Sensor," IEEE Sens. J., vol. 15, pp. 5087-5094, 2015.

[23] Y. C. Liang and D. Tien, "Development of a portable electrical capacitance tomography system," IECON 2011 37th Annu. Conf. IEEE Ind. Electron. Soc., pp. 2634-2638, 2011.

[24] A. Shafquet, et al., "Application of Electrical Capacitance Tomography on Single-Plane Sensor Measurement," 2013 IEEE International Conference on Smart Instrumentation, Measurement and Applications (ICSIMA), 2013, pp. 26-27.

[25] Z. Wang and W. Zhang, "Simulation study of Electrical Capacitance Tomography," 2011 Second Int. Conf. Mech. Autom. Control Eng., 2011, pp. 7682-7685.

[26] Y. Li and W. Yang, "Image reconstruction by nonlinear Landweber iteration for complicated distributions," Meas. Sci. Technol., vol. 19, pp. 94014, 2008.

[27] W. Yang, "Design of electrical capacitance tomography sensors," Meas. Sci. Technol., vol. 21, pp. 42001, 2010.

[28] Z. Ren, et al., "A Miniature Two - plate Electrical Capacitance Tomography Sensor," IEEE Sensors Journal, vol. 15(5), 2015.

[29] W. Yang, "Key issues in designing capacitance tomography sensors," Proc. IEEE Sensors, pp. 497-505, 2006. 